\title{
3D EDS Applications Using Destructive and Non-Destructive FIB-Based Techniques
}

\author{
Neal Magdefrau ${ }^{1}$, Julie Wittenzellner ${ }^{1}$, Daniel Goberman ${ }^{1}$, Richard McLaughlin ${ }^{2}$ and Sreenivas Bhattiprolu ${ }^{2}$ \\ ${ }^{1 .}$ United Technologies Research Center, East Hartford, CT 06108 \\ 2. Oxford Instruments America, Inc., Concord, MA 01742
}

The introduction of improved, large area silicon drift detector (SDD) technology for energy dispersive spectrometry (EDS) along with advances in focused ion beam (FIB) techniques provides for a unique opportunity to perform chemical analysis of fine structures in 3-D. The improvements in data collection efficiency, increased count rates and better computing power enables routine collection of 3D EDS datasets for materials science applications.

United Technologies Research Center (UTRC) and Oxford Instruments America, Inc. have recently begun collaborating in the area of 3D EDS to show how these techniques can be used more routinely for industrially relevant materials systems. The hardware being utilized is a state-of-the-art FEI Helios Nanolab 600 dual beam FIB equipped with a retractable backscattered detector, retractable STEM detector, charge neutralization and 5 GIS systems ( $\mathrm{Pt}$ deposition, $\mathrm{C}$ deposition, $\mathrm{W}$ deposition, $\mathrm{XeF}_{2}$ insulator etch, selective carbon mill). The instrument is also equipped with an Oxford $80 \mathrm{~mm}^{2} \mathrm{X}-\mathrm{Max}^{\mathrm{N}} \mathrm{SDD}$ detector which allows for highly efficient collection of generated x-rays.

In addition to state-of-the-art hardware, Oxford instruments has recently released proprietary LayerProbe $($ C software for non-destructive chemical analysis of complex, multi-layered systems. This non-destructive technique enables lateral chemical analysis resolution of $200 \mathrm{~nm}$ on layer thicknesses which range from $2 \mathrm{~nm}-2000 \mathrm{~nm}$ thick. This non-destructive technique can be validated by destructive techniques such as Auger spectroscopy and FIB serial sectioning. The iterative use of destructive and non-destructive techniques can potentially be used for further understanding and improving on current 3D-EDS techniques.

In this talk, we will show examples using LayerProbe $C$, Auger spectrometry and 3D-EDS to show how the techniques can complement each other. 3D-EDS data of multilayered samples has been collected using dual beam FIB and reconstructed using an Avizo Fire software package. We will conclude the talk with comments on strengths and limitations of each technique and discuss plans for future work in using these findings to further improve both non-destructive (LayerProbeC) and destructive (3D EDS, Auger, XPS) techniques. 


\section{Data acquisition}



Figure 1. Schematic diagram of a data acquisition for 3D-EDS analysis collected using a dual beam FIB.

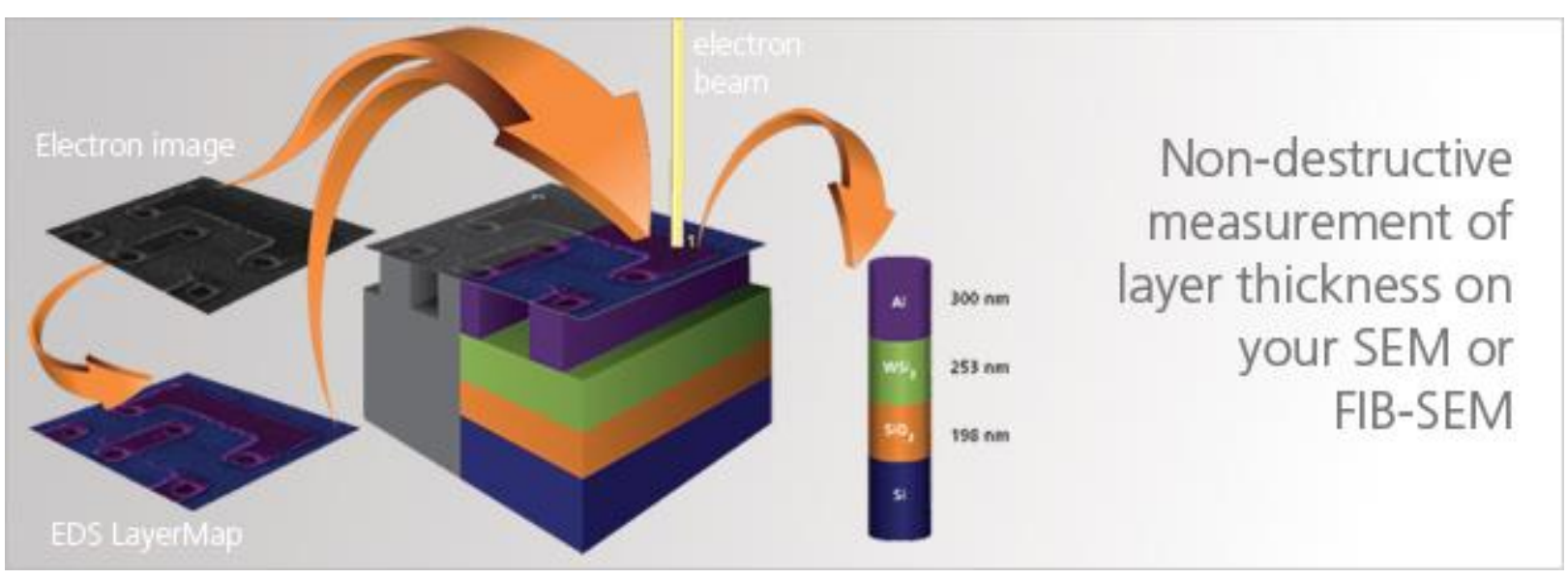

Figure 2. A schematic diagram of Oxford Instrument's non-destructive LayerProbe $\mathbb{C}$ software. 